\title{
Automation in Nuclear Cardiology: Time for Flurpiridaz to Join the Club
}

\author{
Ahmed Aljizeeri, MBBS, ${ }^{\mathrm{a}, \mathrm{b}, \mathrm{c}}$ Firas Al Badarin, MD, MSc, ${ }^{\mathrm{d}}$ \\ and Mouaz H. Al-Mallah, MD, $\mathrm{MSc}^{\mathrm{e}}$ \\ a King Abdulaziz Cardiac Center, Riyadh, Kingdom of Saudi Arabia \\ b King Saud bin Abdulaziz University for Health Sciences, Riyadh, Kingdom of Saudi Arabia \\ c King Abdullah International Medical Research Center, Riyadh, Kingdom of Saudi Arabia \\ d Cleveland Clinic Abu Dhabi, Abu Dhabi, UAE \\ e Houston Methodist DeBakey Heart \& Vascular Center, Houston Methodist Hospital, Houston, \\ TX
}

Received Sep 20, 2020; accepted Sep 21, 2020

doi: $10.1007 /$ s12350-020-02421-9

\section{See related article, pp. 698-708}

Positron emission tomography (PET) is currently the most accurate modality for myocardial perfusion assessment in patients with known or suspected coronary artery disease (CAD). ${ }^{1}$ Notwithstanding its robust diagnostic and prognostic performance as an imaging technology, utilization of PET for myocardial perfusion imaging (MPI) remains low, with widespread adoption likely hampered by many factors, including limited access to PET radiotracers. Most PET radioisotopes have a short half-life, and therefore cannot be obtained from regional radio-pharmacies. Additionally, most laboratories performing PET MPI do not have access to an on-site cyclotron, which makes utilization of ${ }^{13} \mathrm{~N}$ ammonia or ${ }^{15} \mathrm{O}$-water (both cyclotron-produced PET tracers) for perfusion assessment logistically challenging $^{2}$. Lastly, whereas relying on ${ }^{82} \mathrm{Rb}$ generator may improve tracer availability, the cost of the generator remains prohibitive for many low-volume laboratories ${ }^{3}$. Therefore, there currently exists a need for newer PET radiotracers with superior kinetic properties (half-life, first-pass extraction rate, positron range, etc.) that will not only improve tracer availability, but also avoid the

Reprint requests: Mouaz H. Al-Mallah, MD, MSc, Houston Methodist DeBakey Heart \& Vascular Center, Houston Methodist Hospital, 6550 Fannin Street, Smith Tower - Suite 1801, Houston, TX 77030; mouaz74@gmail.com

J Nucl Cardiol 2022;29:709-11.

$1071-3581 / \$ 34.00$

Copyright (C) 2020 American Society of Nuclear Cardiology. pitfalls of current tracers and allow performance of PET MPI in conjunction with exercise.

Flurpiridaz, an ${ }^{18}$ F-labeled PET radioisotope, has several favorable tracer characteristics that was introduced with the potential to fill the gaps with current tracers. ${ }^{4}$ Due to its high affinity to mitochondrial complex-1, Flurpiridaz has a high first-pass extraction rate, and therefore may be suited for more accurate quantification of myocardial blood flow. ${ }^{5}$ Furthermore, with a half-life of 110 minutes, Flurpiridaz can be made available in unit doses from regional sources, increasing tracer availability and, more importantly, allowing performance of PET MPI with exercise stress. Early studies have demonstrated the safety and tolerability of Flurpiridaz in addition to superior image quality and diagnostic performance in comparison to Tc-99m single photon emission computed tomography (SPECT) MPI. ${ }^{6,7}$ More recently, Flurpiridaz PET was found to have superior image quality, lower radiation exposure and improved detection of severe coronary stenoses compared with Tc-99m SPECT, especially in women, obese patients and those undergoing pharmacologic stress. $^{8}$

So, is the best we can hope for with a novel agent and 'ideal tracer characteristics' to outperform Tc-99m in identifying patients with obstructive epicardial coronary disease? Is it possible to further augment the diagnostic yield of PET MPI studies performed with Flurpiridaz? The study by Packard and colleagues in this issue of the Journal examines the value of adding automated perfusion quantitation to PET MPI scans performed with Flurpiridaz in patients with suspected CAD. ${ }^{9}$ Exploiting the strengths of machine learning and automated quantitation in analyzing the wealth of data 
available in an imaging study is a promising concept. This has been examined with radionuclide perfusion studies, and also with other imaging modalities, with the aim of improving diagnostic and prognostic yield of studies. ${ }^{10-14}$ The authors in the current study tested the diagnostic accuracy of an automated system for quantification of perfusion defects against core laboratory interpretation by three expert readers with invasive coronary angiography as the reference standard, using data from recent phase-III Flurpiridaz clinical trial. ${ }^{8}$ First, they obtained the normal radiotracer quant in each coronary territory and the global left ventricular myocardium from 40 patients with no CAD. Then, they obtained the lower limits of normal quant distributions and the standard deviation that fits the best diagnostic accuracy using receiver operating characteristic (ROC) curves to detect $\geq 50 \%$ and $\geq 70 \%$ coronary artery stenosis from 45 patients with exercise stress test and 45 patients with pharmacological stress test. The diagnostic accuracy of the automated system then was examined in 548 patients. The diagnostic accuracy for $\geq 50 \%$ and $\geq 70 \%$ coronary artery stenosis of both the visual and automated read was similar, around $70-73 \%$, for the entire cohort and pharmacological stress test. The diagnostic accuracy was lower, $65-68 \%$, for exercise stress test for both visual and automated reads. There were no significant differences between both methods in the individual coronary artery except for $\geq 50 \%$ the left coronary artery stenosis where the sensitivity has improved $39 \%$ to $64 \%$ by automation, however, the specificity significantly decreased from $88 \%$ to $69 \%$. The interobserver agreement was betted with automated methods in both normal and abnormal system.

Although the method employed by the authors is not entirely novel, it has few merits. They standardized the normal limits and what is considered as abnormal in both exercise and pharmacological stress test. In addition, they validated their results in a different population. They have also tested the system in a wide range of patients including normal subjects and patients with single, two and three-vessel disease who were prospectively randomly selected for phase-III clinical trial of Flurpiridaz from multiple centers. However, it is understood the analysis for the current study was retrospective. They have also extensively studied the interobserver agreement in both methods. It also adds to the growing body of literature supporting the future use of the automated analysis of relative perfusion in nuclear myocardial perfusion imaging. ${ }^{15-18}$ These studies help to standardize the assessment of severity and extent of ischemia by quantification. This provides uniform measure of ischemia to follow patients on medical therapy and consider further treatment options. The conclusions of the study have to be interpreted in the context of the study limitations which were addressed by the authors.

Although we concur with the authors optimistic conclusion about the use of automated quantification of ischemia, we should be vigilant for the phantom of the opera. Despite the promising advantages of Flurpiridaz, the diagnostic characteristics are a little disappointing. The sensitivity and specificity for the global myocardial ischemia and individual coronary artery territory ischemia are much lower than other PET tracers. Whether this is an inherent limitation of the Flurpiridaz or related to the recruitment of lower risk patients in phase-III trials is not clear. The diagnostic accuracy of Flurpiridaz was based on assessment of relative perfusion imaging. Hence, the diagnostic performance may improve if other MPI variables such as myocardial blood flow, coronary flow reserve, ejection fraction reserve and transient ischemic dilation are taken into account. Furthermore, the diagnostic accuracy was evaluated against invasive coronary angiography and not invasive functional testing such as invasive fractional flow reserve. Another interesting finding is the low diagnostic accuracy in the left circumflex territory and whether this would be a limitation in the routine use of Flurpiridaz in routine clinical practice. We must wait for more studies and experience of the real-world practice to answer many of these questions.

Although it is still in its infancy, automated assessment of ischemia has many promising clinical and research applications. It provides a standard assessment of ischemia that can be measured objectively through accurate and reproducible quantification. The diagnostic accuracy of Flurpiridaz is still to be discovered and like Nikola Tesla once said, "Let the future tell the truth, and evaluate each one according to his work and accomplishments',

\section{References}

1. Bateman TM, Dilsizian V, Beanlands RS, DePuey EG, Heller GV, Wolinsky DA. American Society of Nuclear Cardiology and Society of Nuclear Medicine and Molecular Imaging Joint Position Statement on the Clinical Indications for Myocardial Perfusion PET. J Nucl Med. 2016;57:1654-6.

2. Al Badarin F, Aljizeeri A, Almasoudi F, Al-Mallah MH. Assessment of myocardial blood flow and coronary flow reserve with positron emission tomography in ischemic heart disease: current state and future directions. Heart Fail Rev. 2017;22:441-53.

3. Al-Mallah MH, Sitek A, Moore SC, Di Carli M, Dorbala S. Assessment of myocardial perfusion and function with PET and PET/CT. J Nucl Cardiol. 2010;17:498-513.

4. Yu M, Nekolla SG, Schwaiger M, Robinson SP. The next generation of cardiac positron emission tomography imaging agents: Discovery of flurpiridaz F-18 for detection of coronary disease. Semi. Nucl Med. 2011;41:305-13. 
5. Maddahi J. Properties of an ideal PET perfusion tracer: New PET tracer cases and data. J Nucl Cardiol. 2012;19:S30-7.

6. Maddahi J, Czernin J, Lazewatsky J, Huang SC, Dahlbom M, Schelbert H, Sparks R, Ehlgen A, Crane P, Zhu Q, Devine M, Phelps M. Phase I, first-in-human study of BMS747158, a novel 18F-labeled tracer for myocardial perfusion PET: Dosimetry, biodistribution, safety, and imaging characteristics after a single injection at rest. J Nucl Med. 2011;52:1490-8.

7. Berman DS, Maddahi J, Tamarappoo BK, Czernin J, Taillefer R, Udelson JE, Gibson CM, Devine M, Lazewatsky J, Bhat G, Washburn D. Phase II safety and clinical comparison with singlephoton emission computed tomography myocardial perfusion imaging for detection of coronary artery disease: Flurpiridaz F 18 positron emission tomography. J Am College Cardiol. 2013;61:469-77.

8. Maddahi J, Lazewatsky J, Udelson JE, Berman DS, Beanlands RSB, Heller GV, Bateman TM, Knuuti J, Orlandi C. Phase-III clinical trial of fluorine-18 flurpiridaz positron emission tomography for evaluation of coronary artery disease. J Am College Cardiol. 2020;76:391-401.

9. Packard RRS, Cooke CD, Van Train KF, Votaw JR, Sayre JW, Lazewatsky JL, Champagne KM, Orlandi C, Garcia EV, Maddahi J. Development, diagnostic performance, and interobserver agreement of a (18)F-flurpiridaz PET automated perfusion quantitation system. J Nucl Cardiol. 2020. https://doi.org/10.1007/ s12350-020-02335-6.

10. Packard RR, Huang SC, Dahlbom M, Czernin J, Maddahi J. Absolute quantitation of myocardial blood flow in human subjects with or without myocardial ischemia using dynamic flurpiridaz $\mathrm{F}$ 18 PET. J Nucl Med. 2014;55:1438-44.

11. Yoda S, Nakanishi K, Tano A, Hori Y, Suzuki Y, Matsumoto N, Hirayama A. Usefulness of automated assessment of nuclear cardiology for prediction of major cardiac events in Japanese patients with known or suspected coronary artery disease: Comparison with conventional visual assessment in a large-scale prognostic study. J Cardiol. 2014;64:395-400.

12. Betancur J, Otaki Y, Motwani M, Fish MB, Lemley M, Dey D, Gransar H, Tamarappoo B, Germano G, Sharir T, Berman DS,
Slomka PJ. Prognostic value of combined clinical and myocardial perfusion imaging data using machine learning. JACC Cardiovasc. Imaging. 2018;11:1000-9.

13. Dekker M, Waissi F, Bank IEM, Lessmann N, Išgum I, Velthuis BK, Scholtens AM, Leenders GE, Pasterkamp G, de Kleijn DPV, Timmers L, Mosterd A. Automated calcium scores collected during myocardial perfusion imaging improve identification of obstructive coronary artery disease. Int J Cardiol Heart Vasculat. 2020;26:100434.

14. Scannell CM, Veta M, Villa ADM, Sammut EC, Lee J, Breeuwer M, Chiribiri A. Deep-learning-based preprocessing for quantitative myocardial perfusion MRI. J Magn Reson Imaging. 2020;51:1689-96.

15. Arsanjani R, Xu Y, Hayes SW, Fish M, Lemley M Jr, Gerlach J, Dorbala S, Berman DS, Germano G, Slomka P. Comparison of fully automated computer analysis and visual scoring for detection of coronary artery disease from myocardial perfusion SPECT in a large population. J Nucl Med. 2013;54:221-8.

16. Berman DS, Kang X, Gransar H, Gerlach J, Friedman JD, Hayes SW, Thomson LE, Hachamovitch R, Shaw LJ, Slomka PJ, Yang LD, Germano G. Quantitative assessment of myocardial perfusion abnormality on SPECT myocardial perfusion imaging is more reproducible than expert visual analysis. J Nucl Cardiol. 2009; 16:45-53.

17. Slomka PJ, Berman DS, Xu Y, Kavanagh P, Hayes SW, Dorbala S, Fish M, Germano G. Fully automated wall motion and thickening scoring system for myocardial perfusion SPECT: Method development and validation in large population. J Nucl Cardiol. 2012;19:291-302.

18. Motwani M, Leslie WD, Goertzen AL, Otaki Y, Germano G, Berman DS, Slomka PJ. Fully automated analysis of attenuationcorrected SPECT for the long-term prediction of acute myocardial infarction. J Nucl Cardiol. 2018;25:1353-60.

Publisher's Note Springer Nature remains neutral with regard to jurisdictional claims in published maps and institutional affiliations. 